\title{
The Joint Impact of Goal Type and Goal Completion Magnitude on Consumer' Post-Goal-Completion Behavior: An Abstract
}

\author{
Junzhou Zhang and Yuping Liu-Thompkins
}

\begin{abstract}
Goal pursuit represents an important psychological mechanism under loyalty programs (Bagchi and Li 2010; Fishbach and Dhar 2005; Lee and Ariely 2006). Although academic research on loyalty programs has examined the extent to which consumers succeed or fail in reward-goal pursuit, insufficient attention has been paid to the consequences of such successes or failures. Addressing this gap, we draw upon research on goal pursuit and counterfactual thinking to examine the effect of goal completion magnitude on individuals' effort toward achieving subsequent goals, and how maintenance versus attainment goal types moderate this relationship. Analyzing flight activities from 5719 members of a major airline's frequent flyer program, we found that (1) individuals who failed (vs. succeeded) at achieving an attainment goal will put more (vs. less) effort into subsequent goal pursuit than individuals who failed (vs. succeeded) at fulfilling a maintenance goal; (2) a linear effect of goal achievement magnitude on future goal pursuit effort under the maintenance goal, such that the more one completed his/her previous goal, the more effort he/she will invest in the subsequent goal cycle; and (3) a reverse U-shaped effect of goal achievement magnitude under the attainment goal, such that substantial goal achievement in the previous goal cycle creates a surprising hampering effect (i.e., close-but-no-cigar effect) on subsequent goal pursuit. Instead, moderate achievement of the previous goal leads to the most effort invested in subsequent goal pursuit. These results and the proposed underlying processes are further explored in a lab experiment. Our research provides new insights for marketing scholars and managers with regard to goal pursuit and loyalty programs.
\end{abstract}

Keywords Loyalty program · Goal pursuit · Counterfactual thinking · Goal ladder · Travel

References Available Upon Request

J. Zhang $(凹) \cdot$ Y. Liu-Thompkins
Old Dominion University, Norfolk, VA, USA
e-mail: jzhan001@ odu.edu; yxxliu@ odu.edu

(C) The Academy of Marketing Science 2020

$\mathrm{S}$. Wu et al. (eds.), Marketing Opportunities and Challenges in a Changing

Global Marketplace, Developments in Marketing Science: Proceedings of the

Academy of Marketing Science, https://doi.org/10.1007/978-3-030-39165-2_81 